\title{
ON 4-LACUNARY SEQUENCES GENERATED BY ERGODIC TORAL ENDOMORPHISMS
}

\author{
KAROL KRZYŻEWSKI
}

(Communicated by J. Marshall Ash)

\begin{abstract}
It is proved that if $\varphi$ is an ergodic endomorphism of $\mathbb{T}^{k}$ and $f$ is a sufficiently regular complex-valued function on $\mathbb{T}^{k}$ with the Haar integral zero, then $\left(f \circ \varphi^{n}\right)$ is a 4-lacunary sequence. Within the class of ergodic toral endomorphisms and sufficiently regular complex-valued functions, applications are given to the convergence of series, a generalization of the ergodic theorem, the existence of solutions of a generalized cohomology equation, and the convergence of moments in the central limit theorem.
\end{abstract}

Throughout the paper $\varphi$ is a continuous surjective endomorphism of the $k$-dimensional torus $\mathbb{T}^{k}=\mathbb{R}^{k} / \mathbb{Z}^{k}$. There exists a unique invertible matrix $A$ over $\mathbb{Z}$ of degree $k$ such that

$$
\varphi(x)=A x \quad\left(\bmod \mathbb{Z}^{k}\right) .
$$

Let $\mu$ be the probability Haar measure on $\mathbb{T}^{k}$ that may be identified with the Lebesgue measure on $\left[0,1{ }^{k}\right.$. Then $\varphi$ preserves $\mu$. We will assume all the time that $\varphi$ is ergodic with respect to $\mu$. Ergodicity of $\varphi$ is equivalent to no eigenvalue of $A$ being a root of unity.

Throughout the paper $(X, \nu)$ is a probability measure space and the $L^{p}(\nu)$ norm is denoted by $\|\cdot\|_{p, \nu}$, where $1 \leq p \leq+\infty$.

$L^{p}(\mu) \quad(1 \leq p \leq+\infty)$ can be identified with the space of all complex-valued functions on $\mathbb{R}^{k}$ with period 1 with respect to each variable and Lebesgue integrable to the $p$ th power on $\left[0,1\left[^{k}\right.\right.$.

$\|\cdot\|_{p}$ will denote the $L^{p}(\mu)$-norm (resp. $L^{p}\left(\left[0,1\left[^{k}\right)\right.\right.$-norm).

Trig $_{n}$ will be the space of all trigonometric polynomials on $\mathbb{T}^{k}$ of degree at most $n$ in each variable $\left(n \in \mathbb{Z}_{+}\right)$.

$\|\cdot\|$ denotes the natural norm on $\mathbb{R}^{k}$ and on any $\mathbb{C}^{m}$.

For $f \in L^{p}(\mu) \quad(1 \leq p<+\infty)$ the $p$ th modulus of continuity $\omega_{f}^{(p)}$ of $f$ is defined as

$$
\omega_{f}^{(p)}(\delta)=\sup _{\|h\| \leq \delta}\|f(\cdot+h)-f(\cdot)\|_{p}
$$

for $\delta>0$, where $f$ on the right-hand side is the corresponding function on $\mathbb{R}^{k}$.

Received by the editors February 5, 1991 and, in revised form, September 24, 1991.

1991 Mathematics Subject Classification. Primary 42C15; Secondary 28D05.

Research supported by Polish scientific grant RP.I.10. 
For a subset $E$ of $L^{1}(\mu), E_{0}$ denotes the set of all $f \in E$ such that $\mu(f)=$ 0 .

$l^{2}$ denotes the space of all complex sequences summable to the 2 nd power, endowed with the natural norm denoted also by $\|\cdot\|_{2}$.

For

$$
r \in] 1, \min \left(\min _{|\lambda|>1} \sqrt[k+1]{|\lambda|}, \min _{|\lambda|<1} \sqrt[k+1]{|\lambda|^{-1}}\right)[
$$

where $\lambda$ runs over all eigenvalues of $A, L^{4, r}(\mu)$ denotes the set of all $f \in L^{4}(\mu)$ such that

$$
\sum_{n=1}^{\infty} n \omega_{f}^{(4)}\left(r^{-n}\right)<+\infty
$$

The right endpoint of the above interval is well defined by Kronecker's theorem, which states that if all the roots of a monic polynomial with integer coefficients and nonzero constant term are not greater than one in modulus, then they are roots of unity $\left[9\right.$, p. $173 ; 17$, Theorem $2.1(\mathrm{i})$, p. 47]. We skip $\min _{|\lambda|<1} \sqrt[k+1]{|\lambda|^{-1}}$ if undefined.

$L^{4, r}(\mu)$ is a complex Banach space, endowed with the norm

$$
\|f\|_{4, r}=\|f\|_{4}+\sum_{n=1}^{\infty} n \omega_{f}^{(4)}\left(r^{-n}\right) .
$$

The following definition will be needed.

A sequence $\left(f_{n}\right)$ in $L^{4}(\nu)$ is called 4-lacunary if there exists a constant $c \in \mathbb{R}_{+}$such that

$$
\left\|\sum_{j=0}^{n} a_{j} f_{n}\right\|_{4, \nu} \leq c\left\|\left(a_{j}\right)_{0}^{n}\right\|
$$

for any $a_{0}, \ldots, a_{n} \in \mathbb{C}$ and $n \in \mathbb{Z}_{+}$.

In a similar way one defines a $p$-lacunary sequence for $p \in] 2,+\infty[[6, p$. 25]. This terminology comes from the theory of lacunary trigonometric series. Namely, if $\left(\lambda_{n}\right)$ is a sequence of positive integers such that $\inf _{n \geq 0}\left(\lambda_{n+1} / \lambda_{n}\right)>1$, then

$$
\cos 2 \pi \lambda_{0} x, \sin 2 \pi \lambda_{0} x, \ldots, \cos 2 \pi \lambda_{n} x, \sin 2 \pi \lambda_{n} x, \ldots
$$

is $p$-lacunary on $[0,1]$ with the Lebesgue measure for any $p \in] 2,+\infty[[18$, Theorem (8.20), p. 215].

We can now state our main result.

Theorem 1. If $f \in L_{0}^{4, r}(\mu)$ then $\left(f \circ \varphi^{n}\right)$ is a 4-lacunary sequence.

For the proof of Theorem 1 we will need several lemmas.

Lemma 1. There exists a constant $c_{k}>0$ such that for $f \in L^{p}(\mu) \quad(1 \leq p<+\infty)$ and $n \in \mathbb{N}$ there exists $T_{n}(f) \in \operatorname{Trig}_{n}$ such that

$$
\left\|f-T_{n}(f)\right\|_{p} \leq c_{k} \omega_{f}^{(p)}\left(n^{-1}\right) .
$$

Moreover, $\mu\left(T_{n}(f)\right)=\mu(f)$ and $\left\|T_{n}(f)\right\|_{p} \leq\|f\|_{p}$.

The first part of the lemma for $k=1$ is Jackson's theorem [3, Theorem 2.2.1, p. 97]. In the general case the proof is similar. The second part follows from the integral representation of $T_{n}(f)$ and the generalized Minkowski inequality.

The following lemma is [8, Lemma 3] formulated in a slightly stronger form. 
Lemma 2. Let $B$ be a matrix over $\mathbb{Z}$ of degree $k$ and let $V_{1}, V_{2}$ be $B$-invariant subspaces of $\mathbb{R}^{k}$ such that $\mathbb{R}^{k}=V_{1} \oplus V_{2}$ and $B\left|V_{1}, B\right| V_{2}$ have no common complex eigenvalue. Then there exists a constant $c>0$ such that $\rho\left(x, V_{1}\right) \geq$ $c\|x\|^{-\operatorname{dim} V_{1}}$ for any $x \in \mathbb{Z}^{k} \backslash V_{1}$, where $\rho\left(x, V_{1}\right)$ is the distance of $x$ from $V_{1}$ induced by the norm $\|\cdot\|$.

The next lemma is presumably known. It was communicated to me by Professor J. Browkin in answering my question that

Lemma 3. Let $P$ be a monic polynomial with integer coefficients, and let each root of $P$ be not less than 1 in modulus but not a root of unity. Then each root of $P$ is greater than 1 in modulus.

Proof. Assume, to the contrary, that $P$ has a root $a \in S^{1}$. We may assume that $P$ is irreducible over $\mathbb{Z}$. There exists a root $b$ of $P,|b|>1$, since, otherwise by Kronecker's theorem, $a$ would be a root of unity. Since $P$ is irreducible over $\mathbb{Z}$ and, therefore, also over $\mathbb{Q}$, there exists an automorphism $\beta$ of the field $\mathbb{C}$ such that $\beta(a)=b$ [2, Proposition 3, p. 111]. Each such automorphism clearly permutes the roots of $P$. Thereby $\beta(\bar{a})$ is a root of $P$ since so is the conjugate $\bar{a}$ of $a$. We have $\beta(a) \beta(\bar{a})=1$ since $a \bar{a}=1$. Hence $|\beta(\bar{a})|=|b|^{-1}<1$, which contradicts the assumption.

We will state the next lemma in a more general form than is needed. The idea of its proof is that used by Lind in his proof of the exponential rate of mixing of Hölder functions for ergodic toral automorphisms [15, Theorem 6]. The last result, in the more general case of ergodic toral endomorphisms, is contained implicitly in [14, Lemma 5.5] with a different proof.

Lemma 4. For any $p \in \mathbb{N} \backslash\{1\}$ and any $r$ as above there exists $m_{0} \in \mathbb{N}$ such that for any $f_{i} \in L^{p}(\mu), i=1, \ldots, p$, with $\mu\left(f_{j}\right)=0, j=1, p$,

$$
\left|\mu\left(\prod_{j=1}^{p} f_{j} \circ \varphi^{k_{j}}\right)\right| \leq c_{k} \sum_{j=1}^{p} \prod_{\substack{i=1 \\ i \neq j}}^{p}\left\|f_{i}\right\|_{p} \omega_{f_{j}}^{(p)}\left(r^{-\max \left(k_{2}, k_{p}-k_{p-1}\right)}\right),
$$

where $k_{1}, \ldots, k_{p} \in \mathbb{Z}_{+}, 0=k_{1} \leq \cdots \leq k_{p}, \max \left(k_{2}, k_{p}-k_{p-1}\right) \geq m_{0}$, and $c_{k}$ is as in Lemma 1 .

Proof. We will give the proof only for $p=4$. In the general case the proof is similar. Let $f_{i} \in L^{4}(\mu)$ and $k_{i} \in \mathbb{Z}_{+}$for $i=1,2,3,4$ with $\mu\left(f_{j}\right)=0$, for $j=1,4$ and $0=k_{1} \leq k_{2} \leq k_{3} \leq k_{4}$. Put $l=\left[r^{m}\right]+1$, where $m=$ $\max \left(k_{2}, k_{4}-k_{3}\right)$. Then Hölder's inequality for four functions, $\varphi$-invariance of $\mu$, and Lemma 1 imply that the left-hand side of $(*)$ is not greater than

$$
c_{k} \sum_{j=1}^{4} \prod_{\substack{i=1 \\ i \neq j}}^{4}\left\|f_{i}\right\|_{4} \omega_{f_{j}}^{(4)}\left(l^{-1}\right)+\left|\mu\left(\prod_{i=1}^{4} T_{l}\left(f_{i}\right) \circ \varphi^{k_{i}}\right)\right| \text {. }
$$

Therefore it is sufficient to prove that

the second summand of the above sum is equal to zero.

In view of Lemma $1, \mu\left(T_{l}\left(f_{i}\right)\right)=0$ for $i=1,4$. Therefore, for the proof of (1), it is enough to show that if $x_{i} \in \bar{B}\left(0,2 \sqrt{k} r^{m}\right) \cap \mathbb{Z}^{k}$ for $i=1,2,3,4$, 
$x_{j} \neq 0$ for $j=1,4$, and $m \in N, m \geq m_{0}$, where $m_{0} \in \mathbb{N}$ is sufficiently large, then it is impossible that

$$
\sum_{i=1}^{4}\left(A^{*}\right)^{k_{i}} x_{i}=0
$$

where $A^{*}$ is the transpose of $A$ and $m_{0}$ depends only on $\varphi$ and $r$. Assume, to the contrary, that (2) is satisfied. Let $E^{s}, E^{u}$, and $E^{c}$ be the $A^{*}$-invariant subspaces of $\mathbb{R}^{k}$ that correspond to the eigenvalues of $A^{*}$ being less than 1 , greater than 1 , and equal to 1 in modulus, respectively. Then

$$
\mathbb{R}^{k}=E^{s} \oplus E^{u} \oplus E^{c} .
$$

Let $\pi^{s}, \pi^{u}$ be the corresponding projection on $E^{s}, E^{u}$, respectively. It is well known that there exist $c_{1}>0$ and $0<\beta<1$ such that

$$
\begin{aligned}
\left\|\left(A^{*}\right)^{n} v\right\| \leq c_{1} \beta^{n}\|v\| & \text { for } v \in E^{s}, \\
\left\|\left(A^{*}\right)^{-n} v\right\| \leq c_{1} \beta^{n}\|v\| & \text { for } v \in E^{u},
\end{aligned}
$$

where $n \in \mathbb{Z}_{+}$. Moreover, $\beta$ may be any number fulfilling

$$
\max \left(\max _{|\lambda|<1}|\lambda|, \max _{|\lambda|>1}|\lambda|^{-1}\right)<\beta<1,
$$

where $\lambda$ runs over all eigenvalues of $A^{*}$. Therefore $\beta$ can be chosen so that

$$
r<\sqrt[k+1]{\beta^{-1}}
$$

For any $v \in \mathbb{R}^{k}$ let $v^{s}=\pi^{s}(v)$ and $v^{u}=\pi^{u}(v)$.

(I) $k_{2} \geq k_{4}-k_{3}$. (a) $x_{1}^{s} \neq 0$. Let us remark that (2) implies

$$
x_{1}^{s}=-\sum_{i=2}^{4}\left(A^{*}\right)^{k_{i}} x_{i}^{s} .
$$

Since $x_{1} \in \mathbb{Z}^{k} \backslash E^{u} \oplus E^{c}$, Lemma 2 yields

$$
\rho\left(x_{1}, E^{u} \oplus E^{c}\right) \geq c\left\|x_{1}\right\|^{-k} .
$$

There exists $c_{2}>0$ depending only on $\varphi$ such that

$$
\begin{aligned}
& \rho\left(x_{1}, E^{u} \oplus E^{c}\right) \leq c_{2}\left\|x_{1}^{s}\right\|, \\
& \left\|v^{s}\right\| \leq c_{2}\|v\| \text { for } v \in \mathbb{R}^{k} .
\end{aligned}
$$

Then (3) and (6)-(9) imply

$$
c\left(2 \sqrt{k} r^{m}\right)^{-k} \leq 6 \sqrt{k} c_{2}^{2} c_{1} \beta^{m} r^{m},
$$

which, in view of (5), is impossible if $m_{0}$ is sufficiently large.

(b) $x_{1}^{s}=0$. We will first prove that

$$
\left(E^{u} \oplus E^{c}\right) \cap \mathbb{Z}^{k}=E^{u} \cap \mathbb{Z}^{k} .
$$

For this purpose, let $G$ denote the left-hand side of $(10)$ and $\mathfrak{a}$ be a $\mathbb{Z}$-basis of the group $G$ (if $G \neq\{0\}$ ). Then $A^{*}(G) \subset G$. If $B$ is the matrix of $A^{*} \mid G$ relative to $\mathfrak{a}$, then $B$ has integer coefficients and each of its eigenvalues is not less than 1 in modulus but is not a root of unity since $\mathfrak{a}$ is also a basis of 
$\operatorname{Span}_{\mathbb{R}} G \subset E^{u} \oplus E^{c}$. In view of Lemma 3, each eigenvalue of $B$ is greater than 1 in modulus. This implies that $G \subset E^{u}$ since the sequence $\left(\left(A^{*}\right)^{-n} v\right)$ does not converge to zero for $v \in E^{c} \backslash\{0\}$. Thus the proof of (10) is completed.

In view of (2) and (10), we have

$$
\begin{gathered}
\left(A^{*}\right)^{-m} x_{1}=-x_{2}-\left(A^{*}\right)^{k_{3}-k_{2}} x_{3}-\left(A^{*}\right)^{k_{4}-k_{2}} x_{1}, \\
x_{1} \in E^{u} .
\end{gathered}
$$

Conditions (4), (5), and (12) imply that $\left\|\left(A^{*}\right)^{-m} x_{1}\right\|<1$ if $m_{0}$ is sufficiently large. This and (11) give that the right-hand side of (12), being in $\mathbb{Z}^{k}$, is zero. Therefore, $x_{1}=0$. This contradicts the assumption.

(II) $k_{2}<k_{4}-k_{3}$. Equation (2) implies that

$$
x_{4}^{u}=-\sum_{i=1}^{3}\left(A^{*}\right)^{k_{i}-k_{4}} x_{i}^{u} .
$$

Notice that $x_{4} \notin E^{s} \oplus E^{c}$, since otherwise by Kronecker's theorem (see the proof of (10)), $A^{*}$ would have a root of unity as an eigenvalue. Further reasoning is similar to that in (I)(a). One applies Lemma 2 to $E^{s} \oplus E^{c}$ and $x_{4}$ and then uses (4), (5), (13), and the inequalities that correspond to (8) and (9) if one replaces $x_{1}, x_{1}^{s}, E^{u} \oplus E^{c}, v^{s}$ by $x_{4}, x_{4}^{u}, E^{s} \oplus E^{c}, v^{u}$, respectively. Therefore we omit this part of the proof. Thus the proof of the lemma is complete.

The following lemma follows from [7, Theorem 3].

Lemma 5. Let $(X, \nu)$ be a probability measure space and let $\left(f_{n}\right)$ be a sequence of real functions bounded in $L^{4}(\nu)$. Assume that there exists a sequence $(\alpha(n))$ of nonnegative numbers such that

$$
\left|\nu\left(f_{k_{1}} f_{k_{2}} f_{k_{3}} f_{k_{4}}\right)\right| \leq \alpha\left(\max \left(k_{2}-k_{1}, k_{4}-k_{3}\right)\right)
$$

for any $k_{1}, k_{2}, k_{3}, k_{4} \in \mathbb{Z}_{+}, k_{1}<k_{2}<k_{3}<k_{4}$, and $\sum_{n=1}^{\infty} n \alpha(n)<+\infty$. Then $\left(f_{n}\right)$ is a 4-lacunary sequence.

Theorem 1 follows from Lemmas 4 and 5.

Remark 1. In particular, Theorem 1 may be applied to Hölder functions with respect to the distance induced on $\mathbb{T}^{k}$ by the natural distance on $\mathbb{R}^{k}$ or, equivalently, to functions whose corresponding functions on $\mathbb{R}^{k}$ are Hölder with respect to the natural distance on $\mathbb{R}^{k}$. In the case of hyperbolic toral automorphisms and Hölder functions, Theorem 1 follows from Lemma 2 in [10], whose proof uses the technique of Markov partitions. This method can be generalized to cover also the case of hyperbolic toral endomorphisms and Hölder functions [11].

Remark 2. Theorem 1 can be generalized to functions included in $L_{0}^{p, r}(\mu) \cap$ $L^{q}(\mu)$, where $1 \leq p \leq q \leq+\infty, p^{-1}+3 q^{-1}=1$, and the space $L^{p, r}(\mu)$ is defined analogously as $L^{4, r}(\mu)$. The proof requires no essential changes.

From now on $f \in L_{0}^{4, r}(\mu)$ for some $r$, unless otherwise stated. Let

$$
\left(f,\left(a_{k}\right)\right) \stackrel{F_{n}}{\rightarrow} \sum_{j=0}^{n} a_{j} f \circ \varphi^{j} \quad \text { for } n \in \mathbb{Z}_{+} .
$$


Then $\left(F_{n}\right)$ is a sequence of 2-linear continuous operations from $L_{0}^{4, r}(\mu) \times l^{2}$ into $L^{4}(\mu)$. Theorem 3.3 in [1] (see also [7, Theorem 1.3.5; 16, Theorem A]) implies

Corollary 1. (a) If $\left(a_{n}\right) \in l^{2}$, then the series $\sum_{n=0}^{\infty} a_{n} f \circ \varphi^{n}$ converges $\mu$-a.e. and in $L^{4}(\mu)$.

(b) There exists $d \in \mathbb{R}_{+}$such that

$$
\left\|\sup _{n \geq 0}\left|\sum_{j=0}^{n} a_{j} f \circ \varphi^{j}\right|\right\|_{4} \leq d\left\|\left(a_{n}\right)\right\|_{2}\|f\|_{4, r} \quad \text { for any }\left(a_{n}\right) \in l^{2} .
$$

Moreover, (a) and (b) are true if one replaces $\left(f \circ \varphi^{n}\right)$ by any of its permutations. The constant $d$ depends on $\varphi$ and $r$ but neither on $f$ nor the permutation.

From Corollary 1 follows a generalization of the ergodic theorem and the dominated ergodic theorem within the class of ergodic toral endomorphisms and sufficiently regular complex-valued functions.

Corollary 2. Let $\left(k_{n}\right)$ be a sequence of natural numbers with all different terms. Then

(a) $n^{-1} \sum_{j=1}^{n} f \circ \varphi^{k_{j}} \rightarrow 0 \quad \mu$-a.e. and in $L^{4}(\mu)$.

(b) There exists $\bar{d} \in \mathbb{R}_{+}$such that $\left\|\sup _{n \geq 1}\left|n^{-1} \sum_{j=1}^{n} f \circ \varphi^{k_{j}}\right|\right\|_{4} \leq \bar{d}\|f\|_{4, r}$, where the constant $\bar{d}$ depends on $\varphi$ and $r$ but neither on $f$ nor $\left(k_{n}\right)$.

In the proof one uses the identity

$$
n^{-1} \sum_{j=1}^{n} f \circ \varphi^{k_{j}}=S_{n}-n^{-1} \sum_{j=1}^{n-1} S_{j}
$$

for $n \in \mathbb{N}$, where $S_{n}=\sum_{j=1}^{n} j^{-1} f \circ \varphi^{k_{j}}$.

The next lemma can be inferred from [13, Theorem 3] or can be easily proved directly.

Lemma 6. Let $\psi$ be an endomorphism of $(X, \nu)$, and let $h \in L^{2}(\nu)$ be such that $\sum_{n=1}^{\infty} n\left|\nu\left(h \circ \psi^{n} \bar{h}\right)\right|<+\infty$. Then the spectral density $g_{h}$ of $h$ with respect to the dynamical system $(X, \nu, \psi)$ exists, i.e.,

$$
\nu\left(h \circ \psi^{n} \bar{h}\right)=m\left(z^{n} g_{h}(z)\right) \text { for } n \in \mathbb{Z}_{+},
$$

where $g_{h}: S^{1} \rightarrow \mathbb{R}_{+}$and $m$ is the probability Haar measure on $S^{1}$. Moreover, $g_{h}$ is continuous and the sequence

$$
\left(\left\|\sum_{j=0}^{n-1} \lambda^{j} h \circ \psi^{j}\right\|_{2, \nu}^{2}-n g_{h}(\bar{\lambda})\right)
$$

is convergent for any $\lambda \in S^{1}$.

In view of Lemma 4 , the above lemma can be applied to the dynamical system $\left(T^{k}, \mu, \varphi\right)$ and the function $f$.

We will now give an application of Theorem 1 to the existence of solutions of a generalized cohomology equation. 
Corollary 3. Let $\lambda \in S^{1}$. Then the following conditions are equivalent:

(a) there exists a $\mu$-measurable complex-valued function $h$ such that

$$
f=\lambda h \circ \varphi-h \quad \mu \text {-a.e.; }
$$

(b) there exists $h \in L^{2}(\mu)$ such that (**) holds;

(c) the spectral density of $f$ with respect to the dynamical system $\left(\mathbb{T}^{k}, \mu, \varphi\right)$ attains the value zero at $\bar{\lambda}$.

Proof. The proof of $(a) \Rightarrow(b)$ is the same as that in Example 1 of [10]. One uses Theorem 1, Lemma 4 for $p=2$, Lemma 6, and [10, Lemma 1]. In view of Lemma 4 for $p=2$, Lemma 6, and [10, Lemma 1], (b) and (c) are equivalent. This completes the proof of the corollary.

The next lemma is implicitly contained in [6] (see the proof of Theorem 1.2.6, Corollaries 1.3.1 and 1.3.4). For the sake of completeness, we will give the direct proof.

Lemma 7. Let $\left(f_{n}\right)$ be a 4-lacunary sequence of functions on $(X, \nu)$, and let there exist a constant $d>0$ such that

$$
\left\|\sum_{j=0}^{n} a_{j} f_{j}\right\|_{2, \nu} \geq d\left\|\left(a_{j}\right)_{0}^{n}\right\|
$$

for any $a_{0}, \ldots, a_{n} \in \mathbb{C}$ and $n \in \mathbb{Z}_{+}$. If $\left(a_{n}\right)$ is a complex sequence such that

$$
\sup _{n \geq 0}\left|\sum_{j=0}^{k_{n}} a_{j} f_{j}\right|<+\infty \quad \nu \text {-a.e. }
$$

for some increasing sequence $\left(k_{n}\right)$ of nonnegative integers, then $\left(a_{n}\right) \in l^{2}$.

Proof. Assume to the contrary that $\left(a_{n}\right) \notin l^{2}$. Let

$$
g_{n}=\sum_{j=0}^{k_{n}} a_{j} f_{j} /\left\|\sum_{j=0}^{k_{n}} a_{j} f_{j}\right\|_{2, \nu} \quad \text { for } n \geq n_{0},
$$

where $n_{0} \in \mathbb{N}$ is sufficiently large. Then $g_{n} \rightarrow 0 \quad \nu$-a.e. Therefore

$$
\nu\left(A_{n}\right) \rightarrow 1, \quad \text { where } A_{n}=\left\{x \in X:\left|g_{j}\right| \leq n \text { for any } j \geq n_{0}\right\},
$$

for $n \in \mathbb{N}$. Moreover, $\nu\left(\left|g_{n}\right|^{2} \chi_{A_{k}}\right) \rightarrow 0$ for any $k \in \mathbb{N}$, where $\chi_{B}$ is the indicator of a set $B \subset X$. Hence

$$
\nu\left(\left|g_{n}\right|^{2} \chi_{A_{k}^{c}}\right) \rightarrow 1 \text { for } k \in \mathbb{N}
$$

since $\left\|g_{n}\right\|_{2, \nu}=1$ for $n \geq n_{0}$. Applying the Schwarz inequality, we have

$$
\nu\left(\left|g_{n}\right|^{2} \chi_{A_{k}^{c}}\right) \leq\left(\nu\left(A_{k}^{c}\right)\right)^{1 / 2}\left\|g_{n}\right\|_{4, \nu}^{2} \quad \text { for } n \geq n_{0} \text { and } k \in \mathbb{N} .
$$

This and (14) and (15) give a contradiction since $\left(g_{n}\right)$ is bounded in $L^{4}(\nu)$. Thus the lemma is proved.

We will now prove the following: 
Theorem 2. The following conditions are equivalent:

(a) for any complex sequence $\left(a_{n}\right)$, if the series $\sum_{n=0}^{\infty} a_{n} f \circ \varphi^{n}$ converges $\mu$-a.e., then $\left(a_{n}\right) \in l^{2}$;

(b) for any complex sequence $\left(a_{n}\right)$, if $\sup _{n \geq 0}\left|\sum_{j=0}^{k_{n}} a_{j} f \circ \varphi^{j}\right|<+\infty \quad \mu$-a.e. for some increasing sequence $\left(k_{n}\right)$ of nonnegative integers, then $\left(a_{n}\right) \in l^{2}$;

(c) there exists a constant $d>0$ such that

$$
\left\|\sum_{j=0}^{n} a_{j} f \circ \varphi^{j}\right\|_{2} \geq d\left\|\left(a_{j}\right)_{0}^{n}\right\|
$$

for any $a_{0}, \ldots, a_{n} \in \mathbb{C}$ and $n \in \mathbb{Z}_{+}$;

(d) the spectral density of $f$ with respect to the dynamical system $\left(\mathbb{T}^{k}, \mu, \varphi\right)$ is positive;

(e) there are no $\lambda \in S^{1}$ and $h \in L^{2}(\mu)$ such that $(* *)$ is satisfied;

(f) for any complex sequence $\left(a_{n}\right)$; if the series $\sum_{n=0}^{\infty} a_{n} f \circ \varphi^{n}$ converges in $L^{2}(\mu)$, then $\left(a_{n}\right) \in l^{2}$;

(g) for any complex sequence $\left(a_{n}\right)$, if the sequence $\sum_{j=0}^{k_{n}} a_{j} f \circ \varphi^{j}$ is bounded in $L^{2}(\mu)$ for some increasing sequence $\left(k_{n}\right)$ of nonnegative integers, then $\left(a_{n}\right) \in l^{2}$. Proof. (b) $\Rightarrow$ (a) is obvious. (c) $\Rightarrow$ (b) follows from Theorem 1 and Lemma 7. (d) $\Rightarrow$ (c) follows from the inequality

$$
\left\|\sum_{j=0}^{n} a_{j} f \circ \varphi^{j}\right\|_{2}^{2}=m\left(\left|\sum_{j=0}^{n} a_{j} z^{j}\right|^{2} g_{f}\right) \geq\left\|\left(a_{j}\right)_{0}^{n}\right\|^{2} \inf g_{f}
$$

for $a_{0}, \ldots, a_{n} \in \mathbb{C}$ and $n \in \mathbb{Z}_{+}$, where $g_{f}$ is the continuous spectral density of $f$ with respect to the dynamical system $\left(\mathbb{T}^{k}, \mu, \varphi\right)$ (see the remark after Lemma 6). (e) $\Rightarrow$ (d) follows from Corollary 3. For the proof of (a) $\Rightarrow(e)$, let us assume, to the contrary, that there exist $\lambda \in S^{1}$ and $h \in L^{2}(\mu)$ such that $(* *)$ is satisfied. Then it is enough to prove that the series

$$
\sum_{n=1}^{\infty} n^{-1 / 2} \lambda^{n}\left(\lambda h \circ \varphi^{n+1}-h \circ \varphi^{n}\right)
$$

converges $\mu$-a.e. For this purpose notice that

$$
n^{-1} h^{2} \circ \varphi^{n} \rightarrow 0 \quad \mu \text {-a.e. }
$$

by the ergodic theorem applied to the dynamical system $\left(\mathbb{T}^{k}, \mu, \varphi\right)$ and the function $h^{2}$. In view of (16), applying the Abel transformation, it is enough to show that the series

$$
\sum_{n=1}^{\infty}\left(n^{-1 / 2}-(n+1)^{-1 / 2}\right) \lambda^{n+1} h \circ \varphi^{n+1}
$$

converges $\mu$-a.e. For this purpose let us remark that

$$
\text { the sequence }\left(n^{-1} \sum_{j=0}^{n-1} \lambda^{j} h \circ \varphi^{j}\right) \text { converges } \mu \text {-a.e. }
$$


by [5, Theorem 6, p. 675]. In view of (17), applying once more the Abel transformation it is enough to prove that the series

$$
\sum_{n=1}^{\infty}\left(n^{-1 / 2}-2(n+1)^{-1 / 2}+(n+2)^{-1 / 2}\right) \sum_{j=2}^{n+1} \lambda^{j} h \circ \varphi^{j}
$$

converges $\mu$-a.e. This follows from (17) and the inequality

$$
\left|n^{-1 / 2}-2(n+1)^{-1 / 2}+(n+2)^{-1 / 2}\right| \leq n^{-5 / 2} \text { for } n \in \mathbb{N}
$$

$(\mathrm{c}) \Rightarrow(\mathrm{g})$ and $(\mathrm{g}) \Rightarrow(\mathrm{f})$ are obvious. $(\mathrm{f}) \Rightarrow(\mathrm{e})$ follows from the reasoning at the beginning of the proof of $(\mathrm{a}) \Rightarrow(\mathrm{e})$. Thus the proof of the theorem is completed.

Remark 3. Suppose that $\varphi$ is exact with respect to $\mu$. It is easy to see that then in Theorem 2(b) one can replace " $\mu$-a.e." by "on a set of positive $\mu$-measure." Probably the assumption of exactness is superflous. This is so in the case of hyperbolic toral automorphisms and Hölder functions provided that $k_{n}=n$ for $n \in \mathbb{Z}_{+}[10$, Theorem 2]. The above has an extension to the case of hyperbolic toral endomorphisms and Hölder functions [11].

Remark 4. Theorem 2 clearly encompasses, for any $\varphi$, the set of all nontrivial characters.

From now on assume that $f$ is additionally real. If $f \in L_{0}^{2}(\mu)$ and $\omega_{f}^{(2)}(\delta)=$ $O\left(\left(\ln \delta^{-1}\right)^{-\alpha}\right)$ as $\delta \rightarrow 0+$ for some $\alpha>1$, then the sequence of distributions of $g_{n}=n^{-1 / 2} \sum_{j=0}^{n-1} f \circ \varphi^{j}$ converges to the normal distribution $N\left(0, \sigma_{f}\right)$ (possibly degenerate), where

$$
\sigma_{f}^{2}=\mu\left(f^{2}\right)+2 \sum_{n=1}^{\infty} \mu\left(f \circ \varphi^{n} f\right)
$$

[12, Theorem 2; 14, Theorem 5.2]. This [4, Corollary 7, p. 254] and Theorem 1 imply

Corollary 4. If $f \in L_{0}^{4}(\mu)$ and $\omega_{f}^{(4)}(\delta)=O\left(\left(\ln \delta^{-1}\right)^{-\alpha}\right)$ as $\delta \rightarrow 0+$ for some $a>2$, then for any $j=1,2,3$ (resp. $0<\beta<4$ ) the sequence of $j$ th moments (resp. Bth absolute moments) of $g_{n}$ converges to the jth moment (resp. $\beta$ th absolute moment) of $N\left(0, \sigma_{f}\right)$.

Remark 5. In particular, Corollary 4 may be applied to Hölder functions. From [10, Lemma 2] it follows that if $\varphi$ is a hyperbolic toral automorphism and $f$ is a Hölder function, then in Corollary 4 the convergence of moments (resp. absolute moments) holds without any restriction on their order. By a result of [11], this is also true in the case of hyperbolic toral endomorphisms and Hölder functions.

In a subsequent paper of the author, Lemma 5 will be used in proving that sequences generated by some other endomorphisms of probability measure spaces and sufficiently regular complex-valued functions are 4-lacunary.

\section{REFERENCES}

1. G. Bennett, Unconditional convergence and almost everywhere convergence, $\mathrm{Z}$. Wahrsch. Verw. Gebiete 34 (1976), 135-155.

2. N. Bourbaki, Algèbre, Chapitre V, Hermann, Paris, 1950. 
3. P. L. Butzer and R. J. Nessel, Fourier analysis and approximation, Vol. I, One-dimensional theory, Birkhäuser-Verlag, Basel and Stuttgart, 1971.

4. W. S. Chow and H. Teicher, Probability theory, independence, interchangeability, martingales, Springer-Verlag, Berlin, 1978.

5. N. Dunford and J. T. Schwartz, Linear operators, Part I: General theory, Interscience, New York, 1958.

6. V. F. Gaposhkin, Lacunary series and independence functions, Uspekhi Mat. Nauk. 21 (1966), 3-82. (Russian)

7. (1972), 355-365.

8. Y. Katznelson, Ergodic automorphisms of $\mathbb{T}^{n}$ are Bernoulli shifts, Israel J. Math. 10 (1971), 186-195.

9. L. Kronecker, Zwei Sätze über Gleichungen mit ganzzahligen Coefficienten, J. Reine Angew. Math. 53 (1857), 173-175.

10. K. Krzyżewski, On regularity of measurable solutions of a cohomology equation, Bull. Polon. Acad. Sci. Math. 37 (1989), 279-287.

11. _ On the convergence of series generated by continuous maps with canonical coordinates (in preparation).

12. V. P. Leonov, Central limit theorem for ergodic endomorphisms of compact commutative groups, Dokl. Acad. Sci. USSR 135 (1960), 258-261. (Russian)

13. _ On the time dispersion of time-dependent means of a stationary process, Theory Probab. Appl. 1 (1961), 93-101. (Russian)

14. _ Some applications of higher semi-invariants to the theory of stationary processes, Nauka, Moscow, 1964. (Russian)

15. D. A. Lind, Dynamical properties of quasihyperbolic toral automorphisms, Ergodic Theory Dynamical Systems 2 (1982), 49-68.

16. F. Moricz, Moments inequalities and the strong laws of large numbers, $\mathrm{Z}$. Wahrsch. Verw. Gebiete 35 (1976), 299-314.

17. W. Narkiewicz, Elementary and analytic theory of algebraic numbers, Polish Sci. Publ., Warszawa, 1974.

18. A. Zygmund, Trigonometric series, Vol. I, Cambridge Univ. Press, Cambridge, 1959.

Institute of Mathematics, Warsaw University, Banacha 2, 02-097 Warsaw, Poland 